\title{
Multimodal localization and surgery for epileptic spasms of focal origin: a review
}

\author{
Taylor J. Abel, MD, ${ }^{1,2}$ Emma Losito, MD, ${ }^{3}$ George M. Ibrahim, MD, PhD, ${ }^{1}$ Eishi Asano, MD, $\mathrm{PhD},{ }^{4}$ and \\ James T. Rutka, MD, PhD' \\ 'Division of Neurosurgery, The Hospital for Sick Children, Toronto, Ontario, Canada; ${ }^{2}$ Department of Neurological Surgery, \\ University of Pittsburgh, Pittsburgh, Pennsylvania; ${ }^{3}$ Department of Clinical Neurophysiology, APHP, Necker-Enfants Malades \\ Hospital, Paris, France; and 'Departments of Pediatrics and Neurology, Children's Hospital of Michigan, Wayne State University, \\ Detroit, Michigan
}

\begin{abstract}
Epileptic spasms (ES) are a common manifestation of intractable epilepsy in early life and can lead to devastating neurodevelopmental consequences. Epilepsy surgery for ES is challenging because of inherent difficulties in localizing the epileptogenic zone in affected infants and children. However, recent clinical series of resective neurosurgery for ES suggest that not only is surgery a viable option for appropriately selected patients, but postoperative seizure outcomes can be similar to those achieved in other types of focal epilepsy. Increased awareness of ES as a potentially focal epilepsy, along with advances in neuroimaging and invasive monitoring technologies, have led to the ability to surgically treat many patients with ES who were previously not considered surgical candidates. In this study, the authors review the current state of epilepsy surgery for ES. Specifically, they address how advances in neuroimaging and invasive monitoring have facilitated patient selection, presurgical evaluation, and ultimately, resection planning.
\end{abstract}

https://thejns.org/doi/abs/10.3171/2018.6.FOCUS18217

KEYWORDS epilepsy; infantile spasms; seizures; pediatric neurosurgery; development; pediatric neurology

$\mathrm{E}$ PILEPTIC spasms (ES) are a common seizure semiology in young children, characterized by jerking movements that are brief and can occur in clusters. ${ }^{25,36}$ ES usually occur in children at 3-12 months of life, and in such cases are identified as infantile spasms (IS), but they can also occur after the 1st year of life. ${ }^{42}$ Typically, although not uniformly, patients with ES develop other seizure semiologies, such as tonic, tonic-clonic, or focal seizures, by age 3-5 years. ${ }^{21}$ ES can have devastating consequences and are often associated with developmental regression. ${ }^{28}$ First-line medical therapy typically consists of either adrenocorticotropic hormone (ACTH) or vigabatrin..$^{2,28,44}$ Yet, many patients fail these treatment modalities as well as other antiepileptic medications, and continue to have ES and progressive neurodevelopmental regression. In such cases of drug-resistant ES, epilepsy surgery should be considered early in the disease course. ${ }^{18}$ In appropriately selected children (i.e., those with ES of focal or hemispheric origin), resective epilepsy surgery can play a crucial role in achieving seizure freedom ${ }^{8}$ and can provide outcomes similar to those achieved with other forms of medically intractable focal epilepsy. ${ }^{13}$

Identification of the epileptogenic zone (EZ) in ES patients can be challenging, which makes preoperative evaluation in children with drug-resistant ES uniquely difficult. ES semiology often has little lateralizing and no localizing value. ${ }^{36}$ Identification of a focal lesion on MRI facilitates presurgical workup, but in ES the EZ often extends beyond the identifiable lesion. ${ }^{15}$ Furthermore, a large proportion of children with ES have no identifiable lesion and must be evaluated further using other imaging modalities. ${ }^{8,18,30} \mathrm{In}$ both MRI-positive and MRI-negative ES cases, presurgical evaluation often includes either interictal fluorodeoxyglucose PET (FDG-PET) ${ }^{18,20}$ or magnetoencephalography $(\mathrm{MEG})^{41}$ to facilitate identification of the EZ. Ultimately, given that the EZ is often ambiguous after thorough imag-

ABBREVIATIONS ACTH = adrenocorticotropic hormone; DE = depth electrode; $E E G$ = electroencephalography; ES = epileptic spasms; EZ = epileptogenic zone; FDG = fluorodeoxyglucose; ILAE = International League Against Epilepsy; IS = infantile spasms; MEG = magnetoencephalography; SDE = subdural electrode; SEEG = stereoelectroencephalography; VEEG = video EEG.

SUBMITTED April 29, 2018. ACCEPTED June 27, 2018.

INCLUDE WHEN CITING DOI: 10.3171/2018.6.FOCUS18217. 
ing workup, many children require invasive recordings via either subdural grid electrodes $\mathrm{s}^{4,30}$ or stereoelectroencephalography (SEEG) ${ }^{8}$ to delineate the margins and extent of the EZ. Surgical strategies for ES are tailored to the extent of the localized EZ but often involve either multilobar resection or hemispherotomy.

In this article, we review the current state of epilepsy surgery for ES. Specifically, we address how advances in neuroimaging and invasive monitoring have facilitated patient selection, presurgical evaluation, and ultimately, resection planning. In this review, we demonstrate that while challenging, resective epilepsy surgery is a viable treatment strategy for select patients with drug-resistant ES.

\section{Historical Perspective}

Surgery for ES has a relatively new history when compared to surgery for focal epilepsies associated with other seizure semiology. Originally, IS and ES were thought to represent primary generalized epilepsy, and children with ES were not evaluated for surgical intervention. ${ }^{26}$ The description of surgery in clearly lesional ES cases demonstrated the potential role for surgical treatment. In 1979, Branch and Dyken described a 7-month-old infant with ES who underwent resection of a choroid plexus papilloma and subsequently became seizure free. ${ }^{10}$ Subsequently, numerous other case reports emerged demonstrating the efficacy of lesionectomy to treat ES in tumoral ES cases. ${ }^{24,37,43}$ In addition to tumoral ES cases, surgical treatment of porencephalic cysts was also shown to be effective for the treatment ES..$^{40}$ These early cases demonstrated the potential efficacy of resective surgery for ES and led to further research showing benefit of surgery in lesional ES cases. $^{31,33,45}$

With advances in neuroimaging, such as FDG-PET and MEG, pediatric epilepsy teams began attempting surgery in MRI-negative ES cases. The use of FDG-PET to identify epileptic foci in ES patients was first reported in the $1990 \mathrm{~s},{ }^{20}$ though many more cases and case series have now been reported. ${ }^{13,18}$ Subsequently, the use of MEG has been reported to identify the EZ in patients with MRInegative ES. ${ }^{41}$

\section{Patient Selection and Presurgical Evaluation}

Infants and children with ES are considered for epilepsy surgery when ES are deemed to be drug resistant ${ }^{18}$ with the suspicion of a localized origin (some have termed "monofocal"13). ES are considered to be drug resistant when they continue despite treatment with 2 appropriate antiepileptic drugs, including a first-line therapeutic agent-vigabatrin or ACTH. ${ }^{28}$ The focal or nonfocal nature of ES can often be determined by noninvasive evaluation with MRI, PET, and electroencephalography (EEG) ${ }^{3,13}$ Approximately half of children with ES have an identifiable lesion on MRI. For example, a populationbased study performed in Finland identified 42 children with ES and reported the presence of imaging-visible lesions in 26 of 42 children (62\%). ${ }^{27}$ In this study, perinatal insult, brain malformations, and tuberous sclerosis complex were observed in 11,8, and 3 patients, respectively, although it was unclear how many of them had a unifocal lesion. Furthermore, a Canadian study reported that 93 of 216 patients (43\%) visiting their tertiary epilepsy center had focal lesions confined to either the frontal, the centraltemporal-parietal, or the occipital region. ${ }^{34}$ Interestingly, in this study there was an association between lesion location and onset of ES. Occipital lesions were associated with the earliest onset of ES (mean 3.4 months of age), whereas frontal lesions were associated with the latest ES onset (mean 9.8 months). A study of 65 patients with spasms who underwent resective surgery reported that 47 patients had MRI-visible lesions. ${ }^{18}$ Despite the presence of neuroimaging abnormalities in ES patients, further workup is always necessary to confirm the relevance of the neuroimaging abnormality to the electrographic activity. Patients with ES occurring secondary to metabolic disorders, chromosomal abnormalities, diffuse and bilateral brain injury, or diffuse and bilateral brain malformations are unlikely to be candidates for resective epilepsy surgery (although some may still be candidates for palliative surgical interventions). ${ }^{15}$

All ES patients with suspected focal onset should be evaluated for epilepsy surgery by an experienced pediatric epilepsy program. A typical preoperative workflow is depicted in Fig. 1. All patients should undergo video-EEG (VEEG) monitoring and high-resolution MRI, which is the accepted standard. ${ }^{8,13,18,29}$ The essential role of VEEG includes the need to confirm that body jerking events of interest are indeed ES and to rule out the possibility of nonepileptic spells mimicking ES. ${ }^{5}$ Though not necessarily common, video identification of asymmetry in the intensity of body jerking during spasms is useful to lateralize the hemisphere responsible for generating ES. ${ }^{25}$ Scalp EEG changes during spasm events, compared to those associated with focal seizures, are much more subtle, brief, and artifact-prone, whereas interictal epileptiform discharges should be readily identifiable on scalp EEG or MEG in patients with ES.

MRI identification of a focal lesion that is concordant with ictal or interictal epileptiform discharges can guide identification of the EZ. However, the absence of a focal lesion on MRI in the setting of lateralized EEG findings should not discourage further workup. ${ }^{13}$ Some have emphasized the value of FDG-PET imaging in the workup of ES patients, which can facilitate both localization of the $\mathrm{EZ}^{18,20}$ and identification of patients who may have more diffuse epileptogenic pathology $y^{16,17,19}$ (see below). When findings of MRI, VEEG/MEG, and FDG-PET fail to identify the extent of the EZ or when the boundary between the EZ and functionally important cortex is unclear, patients can undergo invasive recordings in the form of either subdural electrode (SDE) recordings, SDE and depth electrode (DE) recordings, or stereoelectroencephalography (SEEG) ${ }^{42}$ Thereby, electrode placement is often guided by multimodal information including FDG-PET ${ }^{18}$ or MEG. ${ }^{30,41}$ Patients with concordant localization of the EZ by MRI, EEG, and FDG-PET may be candidates for localized or hemispheric resections without invasive recordings..$^{13}$

Diagnosis of drug-resistant ES should prompt rapid referral for surgical evaluation. ${ }^{13,18}$ Uncontrolled ES can have devastating neurodevelopmental consequences that 


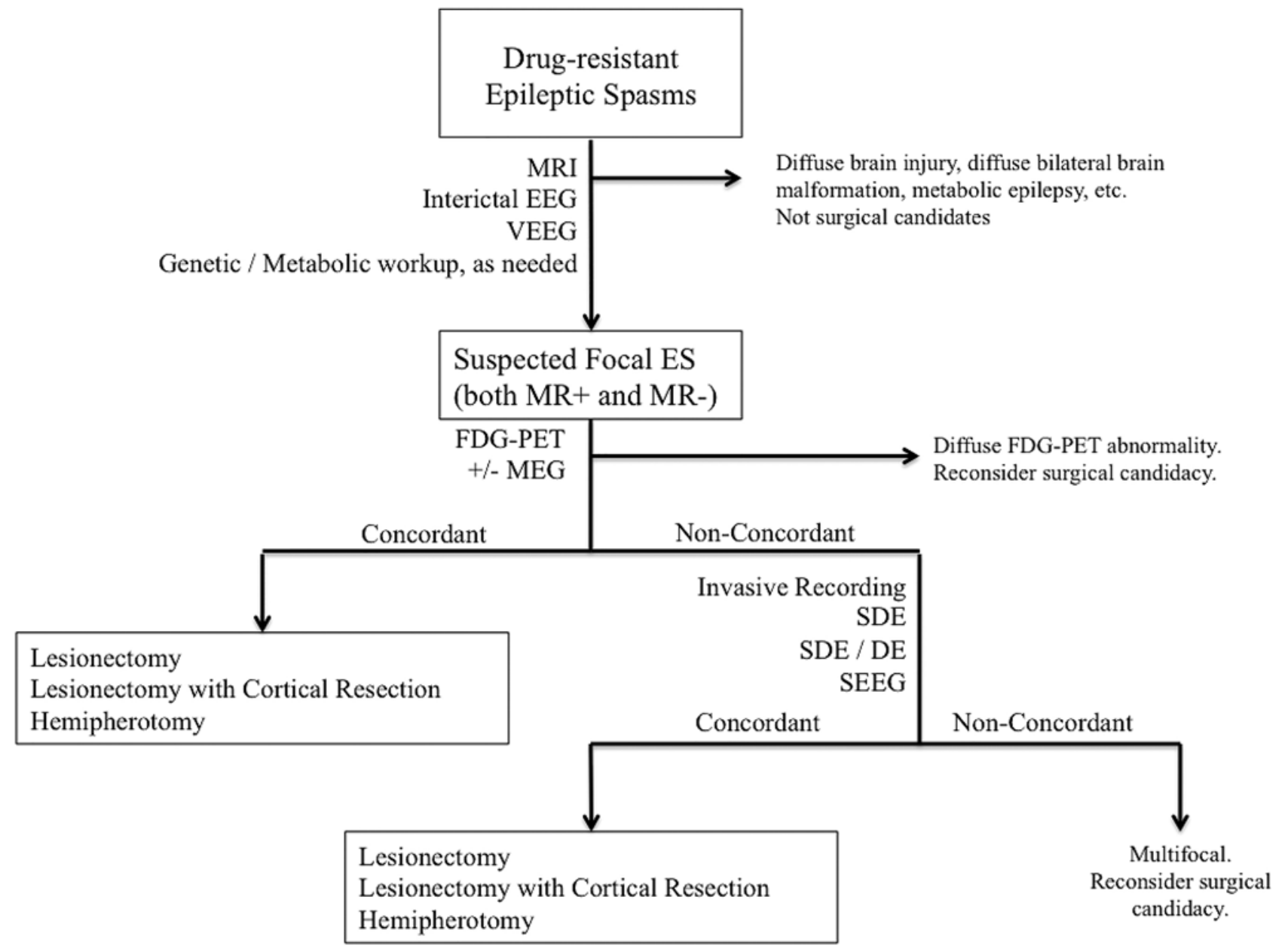

FIG. 1. Schematic representation of presurgical evaluation in infants and children with drug-resistant epileptic spasms (ES).

may be mitigated by surgical intervention. ${ }^{6}$ Furthermore, some data suggest that early surgical intervention improves postoperative seizure-freedom rates. ${ }^{13}$ In a series of children who underwent surgery for ES, Chipaux and colleagues $^{13}$ observed an overall postoperative seizure freedom rate of $74.6 \%$; however, their postoperative seizure freedom rate was $87.9 \%$ in patients who underwent surgery for ES within 36 months of initial diagnosis and $64.7 \%$ in patients who underwent surgery 50 months or longer after initial diagnosis. Furthermore, in a series of ES patients who underwent resective surgery, Chugani and colleagues ${ }^{18}$ found that shorter duration of epilepsy was independently associated with ILAE (International League Against Epilepsy) class I outcome. Early surgery may also influence neurodevelopmental outcome. Asarnow and colleagues ${ }^{6}$ found that patients who underwent early surgery had improved developmental outcomes at 2 years after surgery.

\section{Neuroimaging Techniques for Localizing the EZ in ES}

Given the difficulty in localizing the EZ in children with ES using standard methods (i.e. MRI and video-EEG alone), specialized neuroimaging techniques, such as FDG-PET and MEG, play an integral role in patient selection, accurate localization of foci, and guidance of subsequent intracranial electrode placement. Use of FDG-PET or MEG is particularly important in cases where there is no identifiable lesion on MRI and FDG-PET or MEG may identify or at least lateralize a focal EZ. Table 1 summa-

TABLE 1. Role of PET, MEG, and invasive recordings (SDE or SEEG) for localization of the EZ in patients with ES

\begin{tabular}{lcccc}
\hline \multicolumn{1}{c}{ Authors \& Year } & $\begin{array}{c}\text { No. of } \\
\text { Pts }\end{array}$ & $\begin{array}{c}\text { \% FDG } \\
\text { PET }\end{array}$ & $\begin{array}{c}\% \\
\text { MEG }\end{array}$ & $\begin{array}{c}\text { Chronic Invasive } \\
\text { Recording }\end{array}$ \\
\hline Chipaux et al., 2017 & 68 & 100 & NR & $\begin{array}{c}65 \% ; 3 \text { FO recordings, } \\
\text { 3 SDE/DE, 38 SEEG }\end{array}$ \\
\hline Barba et al., 2016 & 80 & NR & NR & $\begin{array}{c}\text { 30\%; 2 SDE/DE, 22 } \\
\text { SEEG }\end{array}$ \\
\hline limura et al., 2017* & 12 & 66 & 100 & \begin{tabular}{l}
$100 \% ; 12$ SDE \\
\hline Chugani et al., 2015
\end{tabular} \\
\hline
\end{tabular}

$\mathrm{FO}=$ foramen ovale; $\mathrm{NR}=$ not recorded; $\mathrm{Pts}=$ patients.

* Only included ES patients from this article; the authors only considered patients who underwent chronic invasive recordings (SDE). 
rizes recent literature on the role of FDG-PET and MEG in the preoperative workup for ES patients. As observed in recent surgical series, FDG-PET is used frequently in the preoperative evaluation of ES (Table 1). Indeed, some experienced groups consider FDG-PET to be an essential component of the presurgical workup in pediatric ES patients. ${ }^{18}$ MEG can also be helpful in select ES cases..$^{29,30}$

A body of previous work demonstrates a role for FDGPET in the preoperative evaluation of ES patients. In MRInegative ES cases, FDG-PET can identify focal metabolic abnormalities that can aid the localization of the EZ. For example, in a recent case series of surgically treated ES, 12 of $14(85 \%)$ patients with MRI-negative ES demonstrated lateralized, and in sometimes localized, metabolic abnormalities on FDG-PET imaging. ${ }^{18}$ For MRI-negative cases, previous work suggests that when a single region of FDG-PET hypometabolism is identified and is concordant with focal ictal or interictal electrographic abnormality on invasive monitoring, resection of that region would result in seizure control and improvement in developmental delay. ${ }^{15,32}$ Some have also utilized one-stage operations (i.e., without invasive monitoring) in MRI-negative cases where there is a focal abnormality on FDG-PET.

FDG-PET can also play an important role in delineating the extent of the EZ in patients with identifiable lesions in order to determine whether or not invasive recordings are necessary to define the EZ. In lesional cases where FDG-PET abnormalities coincide with the MRI-identified lesion, further workup may not be necessary. In contrast, in lesional cases where the FDG-PET abnormality extends beyond the lesion, it is possible that the EZ may be underestimated by MRI. ${ }^{15}$ This finding may prompt either more extensive resection that is inclusive of the FDG-PET abnormality or invasive recordings that explore the extent of the FDG-PET abnormality and any other regions of hypothesized involvement. Given this utility, some groups have advocated for including FDG-PET as a standard component for presurgical workup in ES patients..$^{13,18}$

FDG-PET may also play a role in identifying patients who may not benefit from surgical intervention. Chugani and colleagues ${ }^{15,17,19,20}$ have described 4 distinct metabolic patterns in cryptogenic IS patients: 1) unifocal (20\%, good surgical candidates), 2) multifocal (65\%), 3) bitemporal $(10 \%)$, and diffuse (5\%). ES patients with multifocal, bitemporal, and diffuse metabolic abnormalities were shown to have distributed or multifocal EZs and were not good candidates for focal resective surgery.

MEG may also play a role in lateralizing or localizing the EZ in patients with focal ES. In a recent report, 9 of 10 patients with ES demonstrated a localized cluster of interictal spike discharges on MEG. ${ }^{29}$ In this series, 4 of the 10 cases were MRI negative, and in 1 of the MRI-negative cases, FDG-PET also demonstrated no abnormality. This suggests that MEG may identify a focal abnormality in MRI-negative ES cases even when FDG-PET does not. In another series, focal MEG clusters were identified in 3 older patients (age 10-14 years) with ES, who subsequently underwent invasive monitoring and subsequent resection with good clinical outcome. ${ }^{41}$ Thus, MEG can provide further localizing information, and may even be useful when no focal abnormality is identified on MRI or FDG-PET.

\section{Invasive Recordings and Electrophysiological Findings in ES}

While FDG-PET and MEG can provide useful localizing data, substantial proportions of patients with ES require invasive monitoring to delineate the $\mathrm{EZ}$ and to determine the boundary between EZ and functionally important areas. Neither FDG-PET nor MEG has replaced invasive monitoring, partly because these imaging modalities essentially localize the regions with interictal abnormalities, whereas the exact margin of the seizure onset zone responsible for generating ES is readily identifiable with invasive recordings. ${ }^{4,38}$ Table 1 summarizes the use of invasive monitoring in recent clinical series of ES surgery. In these series, use of chronic invasive recordings ranged from $30 \%$ to $65 \%$.

In recent series (see Table 1), SDE recordings, a combination of SDE and DE recordings, and SEEG have been employed to characterize the EZ. French and Italian epilepsy teams have favored SEEG recordings, ${ }^{1,12}$ and North American epilepsy teams have favored SDE. ${ }^{18,30}$ However, the ultimate seizure freedom rates achieved by these groups are similar, ranging from $61 \%$ to $83 \%$ Engel class $\mathrm{I}$, as is discussed below in more detail. Whether any particular technique of invasive monitoring is advantageous remains a topic of intense debate..$^{12}$ However, the thin bone of the skull in infants and young children with ES is a relative contraindication for SEEG implantation. ${ }^{1}$ Thus, centers performing ES surgery should have familiarity with SDE techniques even if they typically employ SEEG in invasive monitoring cases.

Invasive recording studies have provided important insight into the pathophysiology of ES. A convergence of evidence suggests that, at least in subsets of patients, ES originate locally in cortex and are characterized by rapid propagation to premotor and motor cortices. ${ }^{4,8,38}$ The ES ictal pattern is often characterized by a diffuse burst of slow waves, with associated low-voltage fast activity, which could superimpose, precede, or follow the slow wave, and are often more focal; this pattern, combined with interictal focal anomalies, can help define the EZ. ${ }^{8,18,38}$ Furthermore, ES can be associated with a focal "leading" spike that precedes a fast-wave burst at $30 \mathrm{~Hz}$ and above at widespread brain regions. ${ }^{4}$ These focal "leading" spikes propagate rapidly and often involve motor or premotor cortex possibly within $100 \mathrm{msec}^{4}$ Asano and colleagues ${ }^{4}$ demonstrated that failure to resect the cortex exhibiting the "leading" spike, if present, was associated with poor surgical outcome in ES patients. Some patients may have a focal seizure preceding or following a cluster of ES, and in such cases, MRI-identified lesions have appeared responsible for both ES and focal seizures. With the sampling rate of recording systems improved over the past decade, Nariai and colleagues ${ }^{38}$ found that the spectral frequencies of "leading" spikes and fast-wave bursts during spasms both reach the range of high-frequency oscillations at $80-200 \mathrm{~Hz}$. Thereby, ictal propagation of high-frequency oscillations to the rolandic cortex was temporally associated with body jerking during ES. ${ }^{4} \mathrm{We}$ have never experienced an incidence of a cluster of ES induced by electrical stimulation via intracranial electrodes 
TABLE 2. Summary of surgical outcomes in recent ES literature

\begin{tabular}{|c|c|c|c|c|c|}
\hline Authors \& Year & $\begin{array}{c}\text { No. of } \\
\text { Pts }\end{array}$ & $\begin{array}{c}\text { Avg Age at } \\
\text { Surgery }\end{array}$ & $\begin{array}{l}\% \text { Invasive } \\
\text { Monitoring }\end{array}$ & Surgery Type & $\begin{array}{l}\% \text { Seizure } \\
\text { Freedom }\end{array}$ \\
\hline Chipaux et al., 2017 & 68 & 4.6 years & 65 & $\begin{array}{l}\text { Focal: } 5 \text { lesionectomy, } 27 \text { lesionectomy + cortical resection, } 7 \\
\text { disconnection } \\
\text { Hemispheric: } 20 \text { hemispherotomy }\end{array}$ & $\begin{array}{l}\text { Focal: } 70 \\
\text { Hemispheric: } 83.3\end{array}$ \\
\hline Barba et al., 2016 & 80 & 5.8 years & 30 & $\begin{array}{l}\text { Focal: } 15 \text { lesionectomy, } 28 \text { lesionectomy + cortical resection, } 15 \\
\text { lobectomy, } 11 \text { corticectomy, } 6 \text { disconnection } \\
\text { Hemispheric: } 4 \text { hemispherotomy, } 1 \text { hemispherectomy }\end{array}$ & 61 \\
\hline limura et al., $2017^{*}$ & 12 & 7.7 & 100 & NR & 75 \\
\hline Chugani et al., 2015 & 65 & 5.1 years & 65 & $\begin{array}{l}\text { Focal: } 13 \text { multilobar resection, } 7 \text { lobectomy, } 6 \text { tuberectomy, } 2 \\
\text { tuberectomy + lobectomy } \\
\text { Hemispheric: } 20 \text { hemispherectomy, } 17 \text { subtotal hemispherotomy }\end{array}$ & 71 \\
\hline
\end{tabular}

Avg $=$ average.

* Only included ES patients from this article; the authors only considered patients who underwent chronic invasive recordings (SDE).

in our practice during the past 2 decades. To the best of our knowledge, there has been no study reporting such an occurrence, whereas focal seizures might happen to be induced by stimulation in patients with ES.? Taken together, we propose that ES of surgical patients reflect a form of secondary generalization of focal seizures, whereas ES are currently classified as "unknown to be focal or generalized" by the ILAE. ${ }^{9}$ Further studies are warranted to determine the difference between the ictal propagation mechanism defining ES and secondary generalized tonicclonic seizures.

\section{Surgical Strategy and Clinical Outcomes}

Table 2 summarizes surgical approaches and clinical outcomes in recent series of resective surgery for ES. As this summary demonstrates, surgical approaches for ES are varied and tailored based on presurgical localization of the EZ in each individual patient. When noninvasive presurgical evaluation demonstrates a clear lesion and no apparent electrophysiological or metabolic abnormality outside the lesion, then a one-stage lesionectomy can be considered. In the setting of a one-stage lesionectomy, acute/intraoperative electrocorticography can play a important role in identifying epileptogenic cortex beyond the margins of the lesion. When electrophysiological or metabolic abnormalities identified during the noninvasive presurgical evaluation extend beyond the lesion, then resection of the lesion and surrounding cortex should be considered. Though there is a range of strategies in this setting, a lesionectomy with corticectomy of the surrounding epileptogenic tissue, more extensive anatomical lobectomy, multilobar resection or disconnection, or even hemispherotomy can be considered. Furthermore, chronic invasive recordings often play an important role to delineate the extent of the EZ beyond the lesion and can be used to plan any of the aforementioned resection strategies.

ES patients with diffuse hemispheric electrographic onset or imaging abnormalities are often considered for hemispherotomy. ${ }^{13,18}$ Yet, resection or disconnection of the somatosensorimotor area may not be in the best interest of ES patients with relatively preserved function of the hand contralateral to the epileptic hemisphere. Iimura and colleagues ${ }^{29}$ recently demonstrated that interictal epileptiform discharges can skip the motor area in patients with ES and that motor-sparing subtotal hemispherotomies result in excellent seizure control with preservation of motor function in patients with multilobar epilepsy, including those with ES..$^{14}$ Therefore, several investigators including Iimura and colleagues have advocated for performing motor-sparing subtotal hemispherotomies in select patients with ES. 14,29,30

It is important to note that despite the challenge of localizing the EZ in patients with ES, postoperative seizurefreedom rates after resective surgery for ES are similar to those achieved in focal epilepsies exhibiting focal semiology. In the recent series summarized in Table 2, postoperative seizure freedom (Engel or ILAE class I outcome) rates ranged from $61 \%$ to $83 \%$, which mirrors similar series of focal epilepsy without ES. ${ }^{22,23,39}$ Complete resection of any MRI-identified lesion and the diagnosed EZ is the most important factor determining favorable outcome. . $^{8,13}$ Furthermore, earlier age at seizure onset and a longer duration of epilepsy before surgery seem to be associated with lower rates of seizure freedom..$^{13}$ In one study of ES surgery, surgery after 3 years of seizure onset was associated with a lower probability of achieving an ILAE class I outcome (i.e., seizure freedom). Furthermore, another study observed a postoperative seizure freedom rate of $87.9 \%$ when surgery was performed within 3 years of first seizure, which dropped to $64.7 \%$ for surgery performed more than 50 months after first seizure..$^{13}$

The neurodevelopmental and behavioral outcomes associated with ES surgery remain poorly understood due to challenges in longitudinal assessment of large numbers of ES patients, heterogeneity of neurodevelopmental status among ES patients, and lack of standardized tests covering a range of developmental periods. However, several studies have found a correlation between early surgical intervention and neuropsychological improvement. ${ }^{18,35} \mathrm{Ca}-$ plan and colleagues ${ }^{11}$ performed a prospective study on the development of nonverbal communication in surgically treated children with medically intractable ES secondary to structural abnormalities and found a significant increase in social interaction postoperatively that was related to the severity of cortical dysplasia in the resected brain tissue. 
Additionally, in the Caplan et al. study neurodevelopmental outcomes were not associated with outcomes related to surgery. This suggests that neurodevelopmental outcomes in ES patients reflect the influence of more complex factors than seizure control alone. Thus, precisely how surgical variables (i.e., timing of surgery and duration of epilepsy prior to surgery) influence neurodevelopmental outcomes remains an unanswered question. Given even the possibility of improved neurocognitive outcome, we recommend rapid referral for surgery in children with ES to determine candidacy for early surgical intervention.

\section{Conclusions}

Resective epilepsy surgery is a viable treatment option for infants and children with drug-resistant ES for which a focal EZ can be identified. As shown in this review, advances in PET and MEG have improved our ability to identify focal epileptogenic abnormalities and facilitate presurgical evaluation of patients with drug-resistant ES. Despite advances in PET and MEG, invasive recordings still play an important role in defining the EZ. Clinical and neurodevelopmental outcomes are superior in patients who undergo early surgical intervention; therefore, ES patients should be referred and evaluated for surgery as early as possible.

\section{References}

1. Abel TJ, Osorio RV, Amorim-Leite R, Mathieu F, Kahane P, Minotti L, et al: Frameless robot-assisted stereoelectroencephalography in children: technical aspects and comparison with Talairach frame technique. J Neurosurg Pediatr 22:37-46, 2018

2. Aicardi J, Mumford JP, Dumas C, Wood S: Vigabatrin as initial therapy for infantile spasms: a European retrospective survey. Epilepsia 37:638-642, 1996

3. Asano E, Chugani DC, Juhász C, Muzik O, Chugani HT: Surgical treatment of West syndrome. Brain Dev 23:668-676, 2001

4. Asano E, Juhász C, Shah A, Muzik O, Chugani DC, Shah J, et al: Origin and propagation of epileptic spasms delineated on electrocorticography. Epilepsia 46:1086-1097, 2005

5. Asano E, Pawlak C, Shah A, Shah J, Luat AF, Ahn-Ewing J, et al: The diagnostic value of initial video-EEG monitoring in children-review of 1000 cases. Epilepsy Res 66:129-135, 2005

6. Asarnow RF, LoPresti C, Guthrie D, Elliott T, Cynn V, Shields WD, et al: Developmental outcomes in children receiving resection surgery for medically intractable infantile spasms. Dev Med Child Neurol 39:430-440, 1997

7. Aungaroon G, Zea Vera A, Horn PS, Byars AW, Greiner HM, Tenney JR, et al: After-discharges and seizures during pediatric extra-operative electrical cortical stimulation functional brain mapping: incidence, thresholds, and determinants. Clin Neurophysiol 128:2078-2086, 2017

8. Barba C, Mai R, Grisotto L, Gozzo F, Pellacani S, Tassi L, et al: Unilobar surgery for symptomatic epileptic spasms. Ann Clin Transl Neurol 4:36-45, 2016

9. Berg AT, Berkovic SF, Brodie MJ, Buchhalter J, Cross JH, van Emde Boas W, et al: Revised terminology and concepts for organization of seizures and epilepsies: report of the ILAE Commission on Classification and Terminology, 20052009. Epilepsia 51:676-685, 2010

10. Branch CE, Dyken PR: Choroid plexus papilloma and infantile spasms. Ann Neurol 5:302-304, 1979

11. Caplan R, Guthrie D, Komo S, Shields WD, Sigmann M:
Infantile spasms: the development of nonverbal communication after epilepsy surgery. Dev Neurosci 21:165-173, 1999

12. Chabardes S, Abel TJ, Cardinale F, Kahane P: Commentary: understanding stereoelectroencephalography: what's next? Neurosurgery 82:E15-E16, 2018

13. Chipaux M, Dorfmüller G, Fohlen M, Dorison N, Metten MA, Delalande O, et al: Refractory spasms of focal onset-a potentially curable disease that should lead to rapid surgical evaluation. Seizure 51:163-170, 2017

14. Chugani HT, Asano E, Juhász C, Kumar A, Kupsky WJ, Sood S: "Subtotal" hemispherectomy in children with intractable focal epilepsy. Epilepsia 55:1926-1933, 2014

15. Chugani HT, Asano E, Sood S: Infantile spasms: who are the ideal surgical candidates? Epilepsia 51 (Suppl 1):94-96, 2010

16. Chugani HT, Conti JR: Etiologic classification of infantile spasms in 140 cases: role of positron emission tomography. $\mathbf{J}$ Child Neurol 11:44-48, 1996

17. Chugani HT, Da Silva E, Chugani DC: Infantile spasms: III. Prognostic implications of bitemporal hypometabolism on positron emission tomography. Ann Neurol 39:643-649, 1996

18. Chugani HT, Ilyas M, Kumar A, Juhász C, Kupsky WJ, Sood S, et al: Surgical treatment for refractory epileptic spasms: the Detroit series. Epilepsia 56:1941-1949, 2015

19. Chugani HT, Shewmon DA, Sankar R, Chen BC, Phelps ME: Infantile spasms: II. Lenticular nuclei and brain stem activation on positron emission tomography. Ann Neurol 31:212-219, 1992

20. Chugani HT, Shields WD, Shewmon DA, Olson DM, Phelps ME, Peacock WJ: Infantile spasms: I. PET identifies focal cortical dysgenesis in cryptogenic cases for surgical treatment. Ann Neurol 27:406-413, 1990

21. Cowan LD, Hudson LS: The epidemiology and natural history of infantile spasms. J Child Neurol 6:355-364, 1991

22. Dwivedi R, Ramanujam B, Chandra PS, Sapra S, Gulati S, Kalaivani M, et al: Surgery for drug-resistant epilepsy in children. N Engl J Med 377:1639-1647, 2017

23. Englot DJ, Breshears JD, Sun PP, Chang EF, Auguste KI: Seizure outcomes after resective surgery for extra-temporal lobe epilepsy in pediatric patients. J Neurosurg Pediatr 12:126-133, 2013

24. Gabriel YH: Unilateral hemispheric ganglioglioma with infantile spasms. Ann Neurol 7:287-288, 1980

25. Gaily EK, Shewmon DA, Chugani HT, Curran JG: Asymmetric and asynchronous infantile spasms. Epilepsia 36:873-882, 1995

26. Gastaut H, Roger J, Ouahchi S, Timsit M, Broughton R: An electro-clinical study of generalized epileptic seizures of tonic expression. Epilepsia 4:15-44, 1963

27. Granström ML, Gaily E, Liukkonen E: Treatment of infantile spasms: results of a population-based study with vigabatrin as the first drug for spasms. Epilepsia 40:950-957, 1999

28. Hancock EC, Osborne JP, Edwards SW: Treatment of infantile spasms. Cochrane Database Syst Rev (4):CD001770, 2008

29. Iimura Y, Jones K, Hattori K, Okazawa Y, Noda A, Hoashi $\mathrm{K}$, et al: Epileptogenic high-frequency oscillations skip the motor area in children with multilobar drug-resistant epilepsy. Clin Neurophysiol 128:1197-1205, 2017

30. Iimura Y, Jones K, Takada L, Shimizu I, Koyama M, Hattori $\mathrm{K}$, et al: Strong coupling between slow oscillations and wide fast ripples in children with epileptic spasms: investigation of modulation index and occurrence rate. Epilepsia 59:544554,2018

31. Iwatani Y, Kagitani-Shimono K, Tominaga K, Okinaga T, Mohri I, Kishima H, et al: Long-term developmental outcome in patients with West syndrome after epilepsy surgery. Brain Dev 34:731-738, 2012 
32. Jonas R, Asarnow RF, LoPresti C, Yudovin S, Koh S, Wu JY, et al: Surgery for symptomatic infant-onset epileptic encephalopathy with and without infantile spasms. Neurology 64:746-750, 2005

33. Kang JW, Rhie SK, Yu R, Eom S, Hong W, Kim SH, et al: Seizure outcome of infantile spasms with focal cortical dysplasia. Brain Dev 35:816-820, 2013

34. Koo B, Hwang P: Localization of focal cortical lesions influences age of onset of infantile spasms. Epilepsia 37:10681071,1996

35. Loddenkemper T, Holland KD, Stanford LD, Kotagal P, Bingaman W, Wyllie E: Developmental outcome after epilepsy surgery in infancy. Pediatrics 119:930-935, 2007

36. Lüders HO: Textbook of Epilepsy Surgery. Boca Raton: CRC Press, 2008

37. Mimaki T, Ono J, Yabuuchi H: Temporal lobe astrocytoma with infantile spasms. Ann Neurol 14:695-696, 1983

38. Nariai H, Nagasawa T, Juhász C, Sood S, Chugani HT, Asano E: Statistical mapping of ictal high-frequency oscillations in epileptic spasms. Epilepsia 52:63-74, 2011

39. Ormond DR, Clusmann H, Sassen R, Hoppe C, Helmstaedter C, Schramm J, et al: Pediatric temporal lobe epilepsy surgery in Bonn and review of the literature. Neurosurgery [epub ahead of print], 2018

40. Palm DG, Brandt M, Korinthenberg R: West syndrome and Lennox-Gastaut syndrome in children with porencephalic cysts: long-term follow-up after neurosurgical treatment, in Niedermeyer E, Degen R (eds): The Lennox-Gastaut Syndrome. New York: Alan R. Liss, 1988, pp 491-526

41. Ramachandrannair R, Ochi A, Imai K, Benifla M, Akiyama $\mathrm{T}$, Holowka S, et al: Epileptic spasms in older pediatric patients: MEG and ictal high-frequency oscillations suggest focal-onset seizures in a subset of epileptic spasms. Epilepsy Res 78:216-224, 2008
42. Ricard-Mousnier B, Dorfmuller G, Fohlen M, Jeanguillaume C, Nguyen S, Delalande O, et al: Late-onset epileptic spasms may be cured by focal cortical resective surgery. Epileptic Disord 14:313-320, 2012

43. Ruggieri V, Caraballo R, Fejerman N: Intracranial tumors and West syndrome. Pediatr Neurol 5:327-329, 1989

44. Vigevano F, Cilio MR: Vigabatrin versus ACTH as first-line treatment for infantile spasms: a randomized, prospective study. Epilepsia 38:1270-1274, 1997

45. Yum MS, Ko TS, Lee JK, Hong S, Kim DS, Kim J: Surgical treatment for localization-related infantile spasms: excellent long-term outcomes. Clin Neurol Neurosurg 113:213-217, 2011

\section{Disclosures}

The authors report no conflict of interest concerning the materials or methods used in this study or the findings specified in this paper.

\section{Author Contributions}

Conception and design: Abel, Losito. Drafting the article: Abel, Losito, Asano, Rutka. Critically revising the article: all authors. Reviewed submitted version of manuscript: Abel, Losito, Ibrahim, Asano. Approved the final version of the manuscript on behalf of all authors: Abel. Study supervision: Rutka.

\section{Correspondence}

Taylor J. Abel: University of Pittsburgh, PA. abelt@pitt.edu. 